\title{
Effective dielectric response of semiconductor composites
}

\author{
Mikhail I. Vasilevskiy and Enrique V. Anda \\ Instituto de Física, Universidade Federal Fluminense, 24210 Niteroi, Rio de Janeiro, Brazil
}

(Received 11 July 1995)

\begin{abstract}
Using a previously proposed diagrammatic approach for the calculation of the renormalized polarizability of spherical inclusions in a homogeneous matrix we obtain an effective dielectric response of a composite of nonpercolating inclusions taking into account a continuous distribution of sizes of the spheres. We apply this theory to semiconductor-doped glasses (SDG) calculating both the electron and phonon responses in the far-infrared and optical spectral regions, respectively, and also to a strongly inhomogeneous semiconductor alloy. For SDG, we compare our calculated results with available experimental data on interband optical absorption and obtain good agreement by choosing the appropriate distribution function of sizes of the spheres. In the case of the $\mathrm{Cd}_{x} \mathrm{Hg}_{1-x} \mathrm{Te}$ alloy, we find an interesting interplay of the composite effects and phononplasmon coupling resulting in a rich structure for the reflectivity spectra. We compare these results to those calculated using another approach, which is widely used for describing the dielectric properties of composites, the self-consistent approximation, and discuss the relation between the two approaches. [S01631829(96)01827-9]
\end{abstract}

\section{INTRODUCTION}

There are several important examples of isotropic inhomogeneous media containing some part of semiconductor material while the rest is nonconducting or has significantly different conductivity. First, inhomogeneities are always present in semiconductor samples. For instance, in dislocation-free silicon so-called $B$ clusters are known, ${ }^{1}$ which are irregularities in the distribution of point defects of the size of a few hundred angstroms formed during growth. Agglomerations $\sim 5 \mu \mathrm{m}$ of electrically active impurities were observed in single crystals of INP. ${ }^{2}$ In some semiconductor alloys, for example $\mathrm{Cd}_{x} \mathrm{Hg}_{1-x} \mathrm{Te}$, components and point defects move easily even at low temperature sometimes producing significant nonuniformity. Formation of clusters of $\mathrm{Hg}$ atoms in $\mathrm{Cd}_{x} \mathrm{Hg}_{1-x}$ Te epilayers during postgrowth cooling or annealing was observed directly. ${ }^{3,4}$ Furthermore, the existence of $p$-type inclusions in an $n$-type matrix was suggested in Refs. 5 and 6 to explain the sharp feature in the temperature dependence of the mobility in bulk samples of this material.

In the case of semiconductor-doped glasses (SDG), the inhomogeneities are introduced intentionally. Semiconductor microcrystallites with sizes of the order of $\leqslant 100 \AA$ (quantum dots) are grown usually in a borosilicate glass host to take advantage of their nonlinear properties due to the quantumconfined effects. $^{7-9}$ Another example of this kind is porous silicon, which has received much attention recently. ${ }^{10}$ However, this last system is anisotropic and will not be considered here.

High-frequency dielectric response of semiconductor structures is of considerable theoretical and practical interest. In the far-infrared (FIR) spectral region, it is governed by free carriers and phonons. FIR spectroscopy is known to be a powerful tool in characterizing bulk semiconductor and epitaxial samples, in particular, to study the free carrier distributions. ${ }^{11}$ It readily provides also the TO and LO phonon frequencies. ${ }^{12}$ As far as SDG is concerned, interband optical absorption experiments usually provide the necessary information about the quantum dot system. ${ }^{8,9}$ In both cases, the probing wave averages over a volume larger than the scale of the inhomogeneities, so what is measured is in fact an effective response of the inhomogeneous medium (composite).

There exists a broad literature regarding the optical properties of isotropic composite materials made of small metallic particles embedded in an insulating matrix (see Refs. 13-18 and references therein). The dielectric response of such systems is usually described in terms of either the effective dielectric medium (DEM) approach or the selfconsistent approximation (SCA). ${ }^{13}$ In the latter case, the matrix is also thought to consist of small spheres, so the components are topologically equivalent. ${ }^{14}$ This approach was extended to describe the transport properties ${ }^{19}$ and the phonon dielectric response $\mathrm{e}^{20}$ of inhomogeneous semiconductors. However, SCA describes a medium in which both phases percolate; i.e., the filling fraction of each of them is between $\frac{1}{3}$ and $\frac{2}{3} \cdot{ }^{13}$ On the contrary, the DEM approximation based on the well-known Maxwell-Garnett (MG) theory ${ }^{21}$ is valid in the low-concentration limit, when inclusions of one material are completely surrounded by a uniform host of the other component. ${ }^{16,18}$ This is just the case of SDG where the filling fraction of the quantum dots does not usually exceed $10 \%$, and also of $\mathrm{Cd}_{x} \mathrm{Hg}_{1-x} \mathrm{Te}$ alloy samples where $p$-type inclusions do not percolate.,

To the best of our knowledge, the DEM approach was not applied to inhomogeneous semiconductor systems, although it provides a natural way of taking account of the statistical distribution of the spheres both in real space and in the space of sizes of the spheres. The effect of the dipolar fluctuations due to the random positions of the spheres was shown to lead to some renormalization of the polarizability compared to the original MG theory where the fluctuations are neglected, and the theory relative to this renormalized polarizability was developed in Ref. 16. However, the averaging problem generated by the dispersion of sizes has not been completely 
resolved, although there have been several attempts to treat it together with the dipolar fluctuations. This is the case of Refs. 15 and 18 where spherical inclusions of two different radii were considered.

In the present paper, we use the diagrammatic formalism proposed in Refs. 16 and 22 to calculate the effective dielectric response, in the dipole approximation, of an isotropic composite with a continuous distribution of sizes of the spheres. In Sec. II, the averaging procedure is performed and the effective renormalized polarizability is obtained. In Sec. III, we apply this theory to the cases of Drude spheres embedded in gelatine, SDG (both the phonon and electron responses in the FIR and optical regions of spectra, respectively, are calculated), and to the inhomogeneous $\mathrm{Cd}_{x} \mathrm{Hg}_{1-x} \mathrm{Te}$ alloy, containing inclusions of strongly different conductivity. In Sec. IV, we discuss the calculated results and also the relation between the DEM approach and SCA. Section $\mathrm{V}$ is devoted to conclusions.

\section{THEORY}

In the DEM approach, the effective dielectric function $\epsilon^{*}$ is related to the (renormalized) polarizability $\alpha^{*}$ of the spheres according to the MG equation: ${ }^{16-18}$

$$
\frac{\epsilon^{*}-\epsilon_{h}}{\epsilon^{*}+2 \epsilon_{h}}=\frac{4}{3} \pi n \alpha^{*},
$$

where $\epsilon_{h}$ is the host dielectric function and $n$ is the concentration of the spheres. Equation (1) was shown to be an approximation of the lowest order in $n \alpha^{*}$. The renormalized polarizability differs from the MG one given by

$$
\alpha(a)=\frac{\epsilon_{s}-\epsilon_{h}}{\epsilon_{s}+2 \epsilon_{h}} a_{0}^{3}
$$

due to local field fluctuations. Putting $\alpha^{*}=\alpha$ one gets the Clausius-Mossotti formula (see, for example, Ref. 23). There is a general equation relating $\alpha^{*}$ to $\alpha$, which was derived elsewhere: ${ }^{16,22}$

$$
\alpha^{*}=\left\langle\alpha \sum_{j} \stackrel{\leftrightarrow}{V}_{i j}^{-1}\right\rangle
$$

where

$$
\begin{aligned}
& \stackrel{\leftrightarrow}{\mathbf{V}}_{i k}^{-1} \stackrel{\leftrightarrow}{\mathbf{V}}_{k j}=\overleftrightarrow{\mathbf{1}} \delta_{i j}, \\
& \stackrel{\leftrightarrow}{\mathbf{V}}_{i j}=\overleftrightarrow{\mathbf{1}} \delta_{i j}-\alpha \Delta \stackrel{\mathbf{T}}{i j}_{i j} \\
& \Delta \stackrel{\mathbf{T}}{i j}_{i j}=\stackrel{\mathbf{T}}{i j}_{i j}-\langle\stackrel{\mathbf{T}}{i j}\rangle
\end{aligned}
$$

and

$$
\stackrel{\leftrightarrow}{\mathbf{T}}_{i j}=e^{-\mathbf{q}(\mathbf{R} i-\mathbf{R} j)}\left(1-\delta_{i j}\right) \boldsymbol{\nabla}_{i} \cdot \boldsymbol{\nabla}_{j} \frac{1}{R_{i j}}
$$

is the dipole-dipole interaction tensor in the quasistatic limit multiplied by the exponential factor, which disappears after taking the long-wavelength limit. In (4) $\overleftrightarrow{\mathbf{1}}$ is the unit tensor, indices $i$ and $j$ run over the spheres and the angular brackets stand for the ensemble average. This average is facilitated by the diagrammatic formalism borrowed from the impurity band theory ${ }^{24}$ and first proposed for this problem in Ref. 16 .

Expanding the inverse of $\overrightarrow{\mathbf{V}}_{i j}$ in series in $\Delta \widehat{\mathbf{T}}_{i j}$ and using the diagram rules defined in Ref. 22 Eq. (3) becomes

$$
\xi \equiv \frac{\alpha^{*}}{\alpha}=0+\varnothing+(\varnothing+\varnothing)+\ldots,
$$

where $\bigcirc=1$, means summation over the spheres, and each line gives the contribution of $\alpha\langle\Delta \stackrel{\mathbf{T}}{i j}\rangle$. The diagram rules ${ }^{16,22}$ imply also taking the longitudinal projection of the contraction of the propagators $\Delta \overrightarrow{\mathbf{T}}_{i j}$, which join the dots $i$ and $j$ and after that the $\mathbf{q} \rightarrow 0$ limit. For example, in the case of the spheres of one size,

$$
\oint=\alpha^{2} n \lim _{\vec{q} \rightarrow 0} \int \vec{e}_{q}\left\langle\Delta \vec{T}_{12}\right\rangle\left\langle\Delta \ddot{T}_{21}\right\rangle \vec{e}_{q} \rho\left(R_{12}\right) d \vec{R}_{12},
$$

where $\mathbf{e}_{q}$ is the unit vector along $\mathbf{q}$ and $\rho\left(R_{12}\right)$ is the binary distribution function of the spheres in real space. Each diagram represents the contribution to the polarizability due to a series of elementary processes in which the fluctuational polarization propagates from sphere $i$ to sphere $j$. Note that expansion (5) contains only irreducible diagrams.

A method of approximate summation of the series (5) was proposed in Ref. 24. Introducing the partial sum of all possible graphs that depart from the open circle and come back to this same circle exactly once, that is

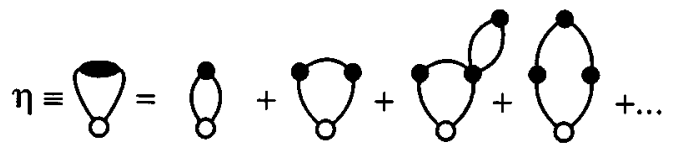

(6) can be rewritten as

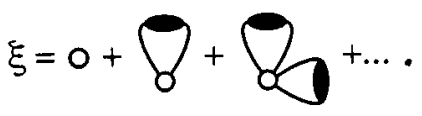

Each partial sum $\eta$ represents the interaction inside a close subset of scatterers. On the other hand, $\eta$ itself can be expressed in terms of $\xi$ :

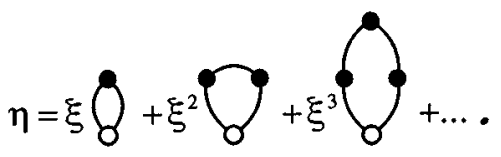

The second term on the right-hand side of (9) represents the contribution of the "second-order remote" spheres, which interact with the reference sphere only through the "firstorder remote" spheres. Thus for a finite system the reasonable approximation of (9) is ${ }^{16}$

$$
\eta=\xi \bigotimes
$$

Equations (8) and (10) enable us to obtain a closed relation for the renormalized polarizability.

Now we have to average (3) over the distribution of sphere's sizes. We do this in two steps. First, for a given sphere of radius $a_{0}$, we average Eq. (8) over all possible radii of all the spheres but the given one. Then, in approxi- 


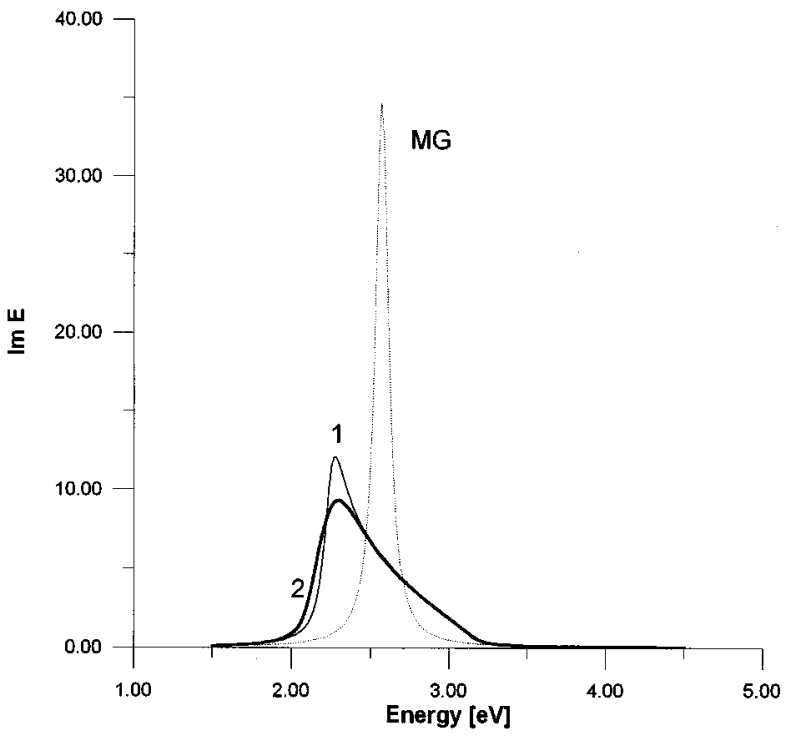

FIG. 1. Imaginary part of $\epsilon^{*}$ as a function of frequency for $\mathrm{Ag}$ spheres in gelatine $f=0.21, \hbar \gamma=0.1 \mathrm{eV}: 1$, spheres of one size; 2 , sizes are uniformly distributed between 0 and $100 \AA$. MG result is also shown for comparison.

mation (10), we have

$$
\langle\rangle_{a i}=\left\langle\xi\left(a_{0}\right) \&\right\rangle_{a_{i}}=\xi\left(a_{0}\right)\langle \&\rangle_{a_{i}} \text {. }
$$

This way we have introduced the same renormalization factor $\xi\left(a_{0}\right)$ depending on the reference sphere size for all the spheres. From (8) and (11) we get

$$
\xi\left(a_{0}\right)=\frac{1}{1-\xi\left(a_{0}\right) \theta},
$$

where

$$
\theta \equiv\langle\rangle_{a i}=8 \pi \alpha\left(a_{0}\right) n \int_{0}^{\infty} d r \int d a \frac{\rho\left(a_{0}, a\right)}{r^{4}} \alpha(a) F(a)
$$

and $F$ is the distribution function of sphere's sizes.

Solving Eq. (12) and performing the remaining average over $\left\{a_{0}\right\}$ we obtain the renormalized polarizability:

$$
\alpha^{*}=\left\langle\xi\left(a_{0}\right) \alpha\left(a_{0}\right)\right\rangle_{a_{0}}=\int d a_{0} \alpha\left(a_{0}\right) \frac{1-\sqrt{1-4 \theta}}{2 \theta} .
$$

Taking the "hole correction" spatial distribution function of the spheres, (13) becomes

$$
\theta=\frac{8}{3} \pi n \alpha\left(a_{0}\right) \int d a \frac{\alpha(a)}{\left(a_{0}+a\right)^{3}} F(a) .
$$

In the case of the spheres of one size, it follows from (14) and (15) that

$$
\alpha^{*}=\frac{2 a^{3}}{\alpha_{0} f}\left(1-\sqrt{1-f \alpha_{0}^{2}}\right),
$$

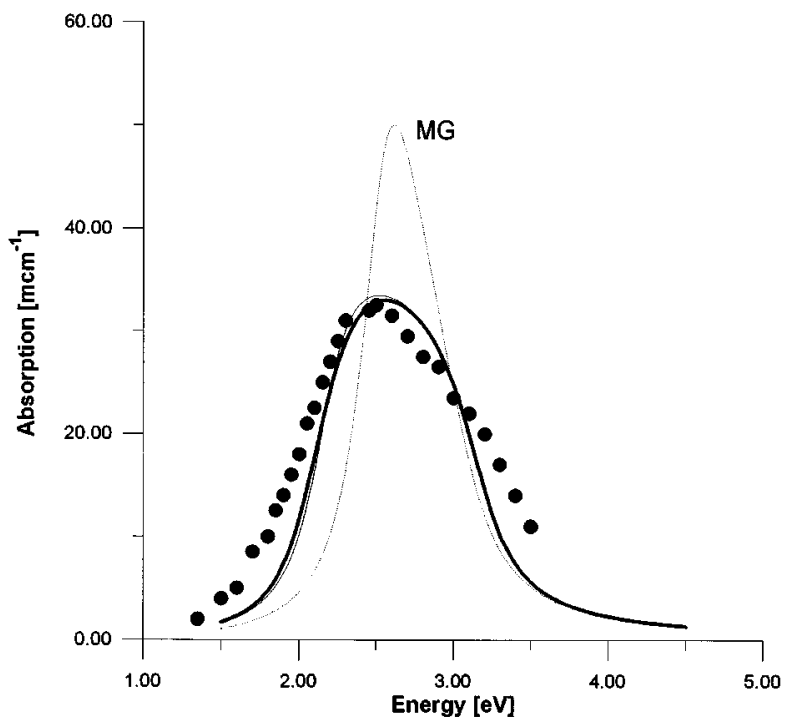

FIG. 2. Absorption coefficient of $\mathrm{Ag}$ particles in gelatine. Curves correspond to those of Fig. 1, but here $\hbar \gamma=0.4 \mathrm{eV}$. Points are experimental data of Ref. 25. The cases 1 and 2 of Fig. 1 are almost not resolved here.

where $f=\frac{4}{3} \pi n a^{3}$ is the filling fraction and $\alpha_{0}=\alpha / a^{3}$. This formula was first derived in Ref. 17.

\section{APPLICATIONS}

\section{A. Drude particles}

In this section we apply formula (14) to a system of $\mathrm{Ag}$ particles embedded in a gelatine host $\left(\epsilon_{h}=2.37\right)$. This system was studied experimentally ${ }^{25}$ and has been used as a touchstone for various theories. ${ }^{15-17,26} \mathrm{We}$ intend to illustrate the size dispersion effect.

Ag particles are a described by a Drude dielectric function

$$
\epsilon_{s}=\epsilon_{\infty}-\frac{\epsilon_{\infty} \omega_{p}^{2}}{\omega^{2}+i \omega \gamma}
$$

with $\epsilon_{\infty}=5.95$ and $\hbar \omega_{p}=3.7 \mathrm{eV}$. In Fig. 1 we show $\operatorname{Im} \epsilon^{*}$ calculated assuming a uniform distribution of sizes within the range $(0 ; 2 \vec{a})$ where $\bar{a}$ is the mean size. The dispersion leads to a decrease of the amplitude and broadening of the peak. The position of the peak is not shifted as it occurs when the filling fraction is changed in the case of the spheres of one size (compare, for example, to Fig. 3 of Ref. 17).

In Fig. 2 we plot the absorption coefficient calculated with and without the dispersion and compare it with experimental data of Ref. 25. Note that within the MG approximation, for the dielectric function of the spheres in the form (17), there is no dependence of the composite properties on sphere's size at all. Within the renormalized MG theory, due to relatively large inhomogeneous broadening, the size dispersion effect is negligible for this system. However, it is satisfying to see that qualitatively reasonable results can be obtained in the 
framework of our approach, which are not worse than those calculated using, for example, SCA. ${ }^{26}$

\section{B. Semiconductor-doped glasses (optical region)}

The effect of crystal-size-dependent inhomogeneous broadening of the absorption line shapes of the optical transitions in SDG can be treated in the same way as for Drude particles if we construct an appropriate dielectric function of a small sphere made of a direct-band-gap semiconductor.

As pointed out in Ref. 27 and recently confirmed experimentally, ${ }^{9}$ correlation effects in microcrystals are unimportant if they are smaller than the excitonic Bohr radius. Here we consider spatially confined electrons and holes as completely decoupled. Then the dielectric function in the optical region can be written (see, e.g., Ref. 28) as follows:

$\boldsymbol{\epsilon}_{s}=\boldsymbol{\epsilon}_{\infty}\left[1+\frac{4 \pi e^{2}}{\boldsymbol{\epsilon}_{\infty} \hbar m_{0}^{2} \omega^{2} V} \sum_{r, r^{\prime}}\left|\mathbf{p}_{r r^{\prime}}\right| 2\left(\frac{1}{\omega_{r r^{\prime}}-\omega}+\frac{1}{\omega_{r r^{\prime}}+\omega}\right)\right]$,

where $V$ is volume, $m_{0}$ is the free electron mass, $\mathbf{p}_{r r^{\prime}}$ is the momentum matrix element between the states $r$ of the conduction band and $r^{\prime}$ of the valence band,

$$
\omega_{r r^{\prime}}=\left(E_{r}^{C}+E_{r^{\prime}}^{V}+E_{g}\right) / \hbar,
$$

$E_{g}$ is the bulk band-gap energy and $r$ stands for $\{n, l, m\}$. The energy levels of electrons and holes are given by

$$
E_{r}^{C, V}=\frac{\hbar^{2} k_{l, n}^{2}}{2 m_{e, h}},
$$

where

$$
k_{l, n}=\frac{\phi_{l, n}}{a}
$$

and $\phi_{l, n}$ is the $n$th root of the spherical Bessel function of order $l$. The matrix element can be evaluated if the envelope function of the confined state varies slowly at the scale of the crystal lattice constant. It is given by

$$
\left|\mathbf{p}_{r r^{\prime}}\right|^{2}=\frac{2}{3} \frac{m_{0}^{2}}{\hbar^{2}} P^{2} \delta_{r r^{\prime}}
$$

where $P$ is known as Kane's momentum matrix element. Using (20) formula (18) can be rewritten as

$$
\epsilon_{s}=\epsilon_{\infty}\left(1+\frac{4 e^{2} P^{2}}{\epsilon_{\infty} \hbar^{3} \omega^{2} a^{3}} \sum_{n, l} \frac{(2 l+1) \omega_{n, l}}{\left(\omega_{n, l}-i \delta\right)^{2}-\omega^{2}}\right),
$$

where we took into account the degeneracy of the levels and introduced the homogeneous broadening parameter $\delta$. Formula (22) with the help of (18) describes the dielectric function of a single semiconductor sphere. We calculated the effective dielectric response of a composite consisting of CdTe spheres embedded in a borosilicate matrix $\left(\epsilon_{h}=3\right)$. The following parameters were taken for CdTe: $\epsilon_{\infty}=7.8$, $P=7.10^{-8} \mathrm{eV} \mathrm{cm}$ for heavy holes, $E_{g}=1.49 \mathrm{eV}$, $m_{\mathrm{hh}}=0.4 m_{0}, m_{\mathrm{lh}}=m_{e}=0.1 m_{0}$, and $\Delta_{1}=1.0 \mathrm{eV} . \hbar \delta=5.10^{-3}$ $\mathrm{eV}$ was used in numerical calculations. Figure 3 illustrates the effect of the size dispersion on the imaginary part of the effective dielectric function. We included five low-energy

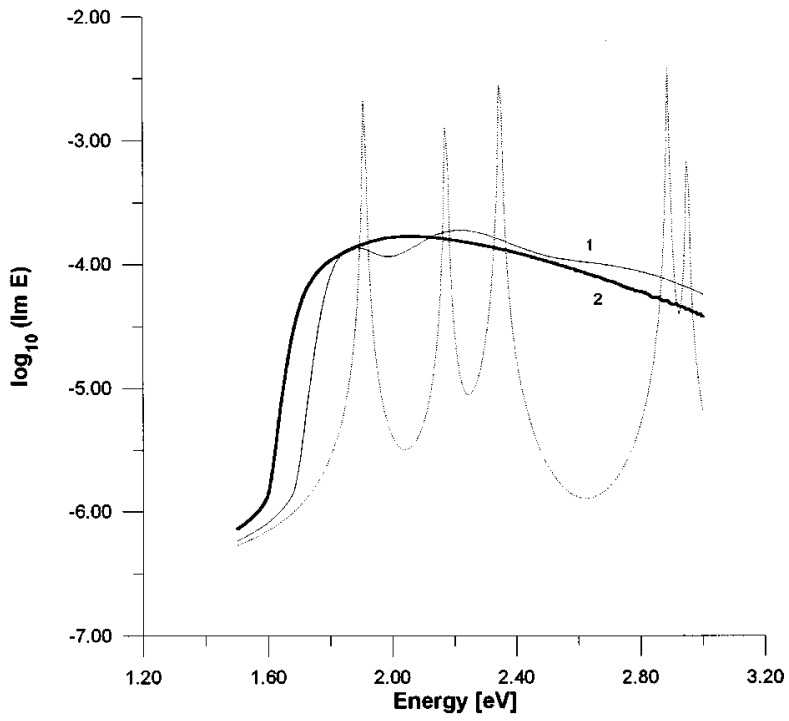

FIG. 3. Calculated dependence of $\operatorname{Im} \epsilon^{*}$ on frequency for CdTe nanocrystals (mean size $35 \AA$ ) in glass without dispersion of sizes (dashed curve) and for Gaussian distribution of sizes with $\sigma-4 \AA$ (1) and $\sigma-8 \AA$ (2).

transitions: $1 S_{\mathrm{hh}}, 1 S_{\mathrm{lh}}, 1 P_{\mathrm{hh}}, 1 P_{\mathrm{lh}}$, and $1 S_{\mathrm{ss}}$, named according to the corresponding valence-band states. If the standard deviation is $\sim 10 \%$ (curve 1 ), some maximuma can be seen in the spectrum although the transitions with close values of the energies cannot be resolved. As $\sigma$ increases further, the spectrum becomes completely smooth (curve 2). We have also fitted linear absorption experimental data ${ }^{29}$ taken from a sample grown by a recrystallization technique described in Ref. 8. The results and the data are shown in Fig. 4. We tried both the Gaussian and the Lifshits-Slyozov (LS) distribution. The latter describes the asymptotic size

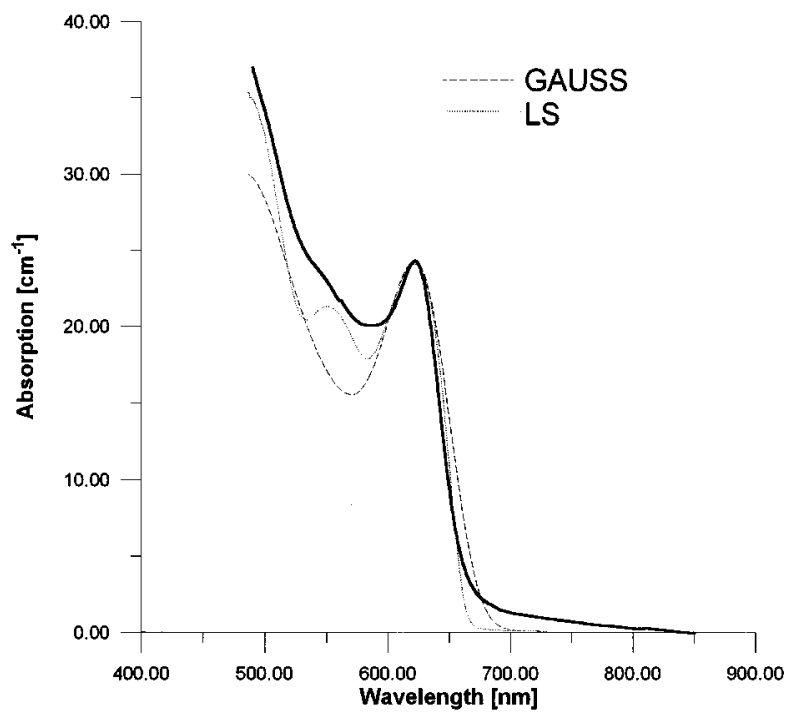

FIG. 4. Absorption coefficient of CdTe-doped glass vs frequency: experimental data (Ref. 29) (full curve) were fitted using a Gaussian distribution (dotted curve, $\bar{a}=31 \AA, \quad \sigma=3 \AA$, $n_{s}=3.8 \times 10^{16} \mathrm{~cm}^{-3}$ ) and LS distribution (dashed curve, $\bar{a}-30 \AA$, $n_{s}-6.45 \times 10^{16} \mathrm{~cm}^{-3}$ ). 


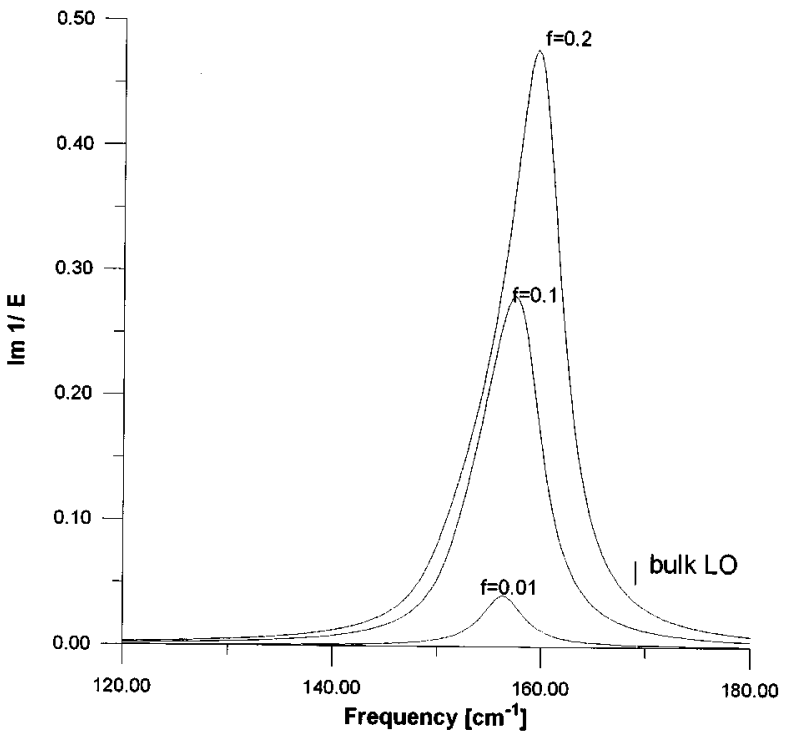

FIG. 5. Imaginary part of the inverse of $\epsilon^{*}$ vs frequency calculated for CdTe-doped glasses of different filling fraction.

distribution of growing particles of a new phase. ${ }^{30}$ We are going to publish more results of this kind elsewhere. ${ }^{29}$ Here we would like to comment that, in general, the LS distribution gives a better fit and it seems more relevant for the growth technique used. ${ }^{8,29}$ The discrepancy that exists in the short-wavelength part of the spectrum may be due to some extra absorption mechanism not included in the model, for instance, the transitions occurring at the $L$ point of the Brillouin zone. ${ }^{31}$ Alternatively, perhaps this can be explained by penetration of the wave functions of electrons and holes into the insulator, ${ }^{9}$ which affects the positions of the levels entering formula (22).

\section{Semiconductor-doped glasses (FIR region)}

Only the semiconductor component produces IR activity due to polar optical phonons. In the vicinity of the phonon reststrahlen band the dielectric function is given by

$$
\epsilon_{s}=\epsilon_{\infty}\left(1+\frac{\omega_{T}^{2}\left(\epsilon_{0}-\epsilon_{\infty}\right)}{\left(\omega_{T}-i \frac{\Gamma_{T}}{2}\right)^{2}-\omega^{2}}\right),
$$

where $\epsilon_{0}$ is the static dielectric constant, $\omega_{T}$ is the bulk phonon frequency, and $\Gamma_{T}$ is phonon damping. One may argue about relevance of the bulk dielectric function (23) for small crystals. Of course, CdTe phonons are confined. The modulus of the LO phonon wave vector is quantized in the same way (20) as for electrons. ${ }^{32}$ However, due to the small dispersion of optical phonons (see, for example, Ref. 33) in CdTe and other $A_{2} B_{6}$ materials, the nonzero but small value of $\mathbf{q}_{\text {min }}$ for the typical microcrystals leads to an insignificant shift of the phonon frequencies. In any case, this quantization of phonons cannot explain the experimentally observed shift $\sim 10 \mathrm{~cm}$ of CdSe LO phonons in SDG downwards from the bulk mode. ${ }^{32}$ As can be seen from Fig. 5, this is rather due to a "composite effect," when features in the spectra of composites are redshifted compared to corresponding longitudi-

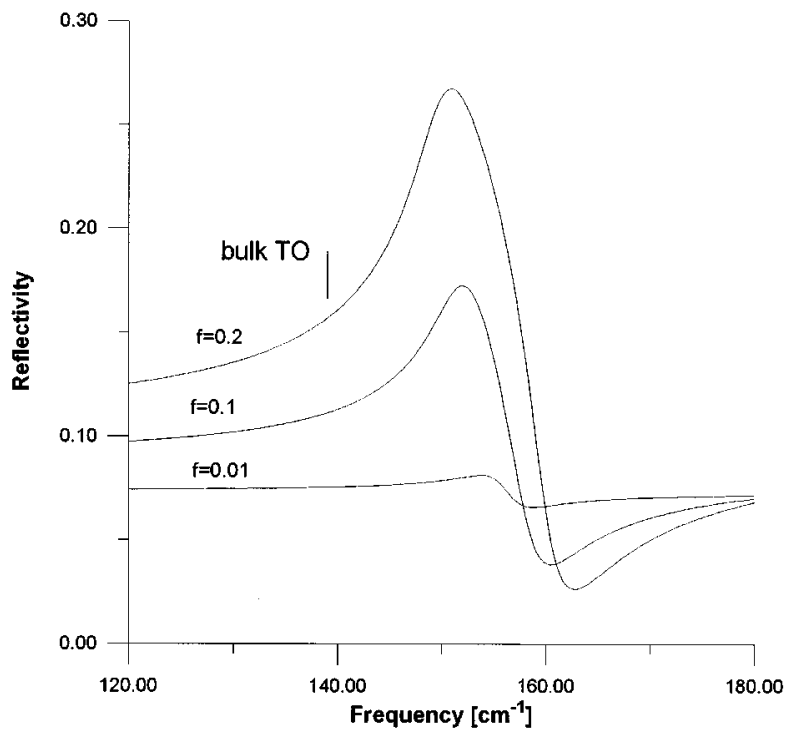

FIG. 6. Calculated normal incidence reflectivity for CdTe-doped glasses of different filling fraction.

nal excitations of an individual sphere. As the filling fraction increases, the shift decreases. The same is true of the TO phonon modes seen in the reflectivity spectra of Fig. 6: the higher the filling fraction, the lower (and closer to the bulk TO frequency) the visible TO mode. Thus, the visible TO and LO composite modes are approaching each other as the filling fraction decreases. This effect can be described by a generalized Lyddane-Sachs-Teller relation if the dielectric function of the system is known. ${ }^{34}$ It was checked experimentally for $\mathrm{ZnSe}$ - diamond composites and modeled within the effective-mass approximation. ${ }^{20}$ Figure 7 illustrates the effect of the size dispersion. Here we have taken into account quantization of phonons discussed above using the calculated dispersion curves for CdTe from Ref. 33. This

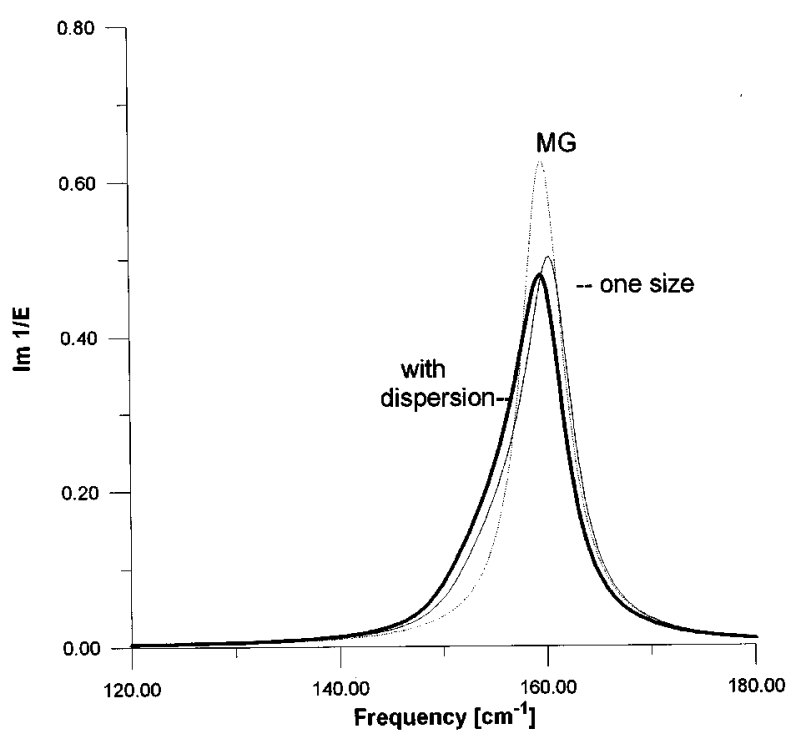

FIG. 7. Same as in Fig. 5 for $f=0.2$ : MG, calculated using the MG polarizability; 1 , spheres of one size but with polarizability; 2, uniform distribution of sizes $35 \pm 20 \AA$. 


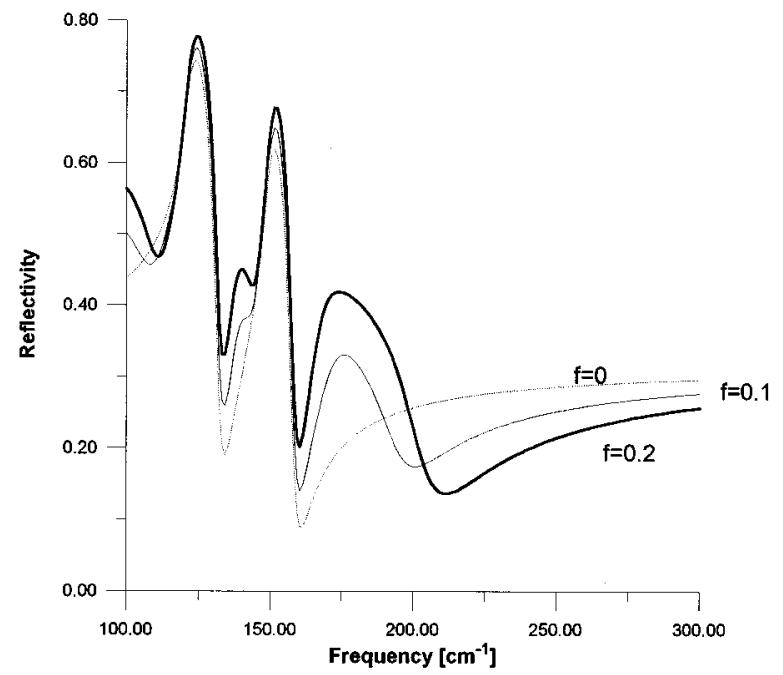

FIG. 8. Normal incidence reflectivity from inhomogeneous CMT for three values of the volume fraction of the metallic inclusions in the dielectric matrix. Splitting of CdTe-like and HgTe-like TO phonons into two modes is seen.

results in a small $2-\mathrm{cm}^{-1}$ shift of the peak compared to the case of the spheres of one size.

\section{Inhomogeneous alloy}

Let us consider a FIR response from an inhomogeneous semiconducting alloy containing regions of two types, which we will call dielectric $(D)$ and metallic $(M)$. For definiteness, we will bear in mind $\mathrm{Cd}_{x} \mathrm{Hg}_{1-x} \mathrm{Te}(\mathrm{CMT})$. Then both types of regions can be described by a FIR dielectric function of the form

$$
\epsilon=\epsilon_{\infty}+\sum_{n=1}^{2} \frac{\rho_{n} \omega_{T_{n}}^{2}}{\omega_{T_{n}}^{2}-\omega^{2}-i \omega \Gamma_{n}}-\frac{\epsilon_{\infty} \omega_{p}^{2}}{\omega^{2}+i \omega \gamma},
$$

where the sum is over phonon resonances at TO frequencies $\omega_{T_{n}}$ since CMT is a two-mode material, with dipole strengths $\rho_{n}$ and damping parameters $\Gamma_{n}$. Explicitly, here we consider both the $D$ ( $p$-type) and $M$ ( $n$-type) regions as $\mathrm{Cd}_{x} \mathrm{Hg}_{1-x} \mathrm{Te}$ of the same composition $x=0.2$, and they differ only by the value of $\omega_{p}$. The phonon parameters were taken as in Ref. 11 . For the $D$ regions we put $\omega_{p}=0$. This is justified because the effective mass of electrons is about two orders of magnitude less than that of holes in CMT.

Calculated results for both possible cases are shown in Figs. 8-10. The normal incidence reflectivity spectra are quite different in the cases of the $M$ inclusions in the $D$ matrix (Fig. 8) and the $D$ inclusions in the $M$ matrix (Fig. 9). For $\omega_{p}=250 \mathrm{~cm}^{-1}$ the resonance frequency for a single $M$ sphere embedded in the $D$ matrix $\sqrt{1 / 3} \omega_{p}$ falls in the region close to the TO frequencies. Consequently, this excitation (let us call it the Maxwell-Garnett excitation) interacts strongly with TO phonons in this case, which results in splitting of the TO reflectivity peaks, as can be seen from Fig. 8 . In the opposite case of Fig. 9, the MG excitation is posed at

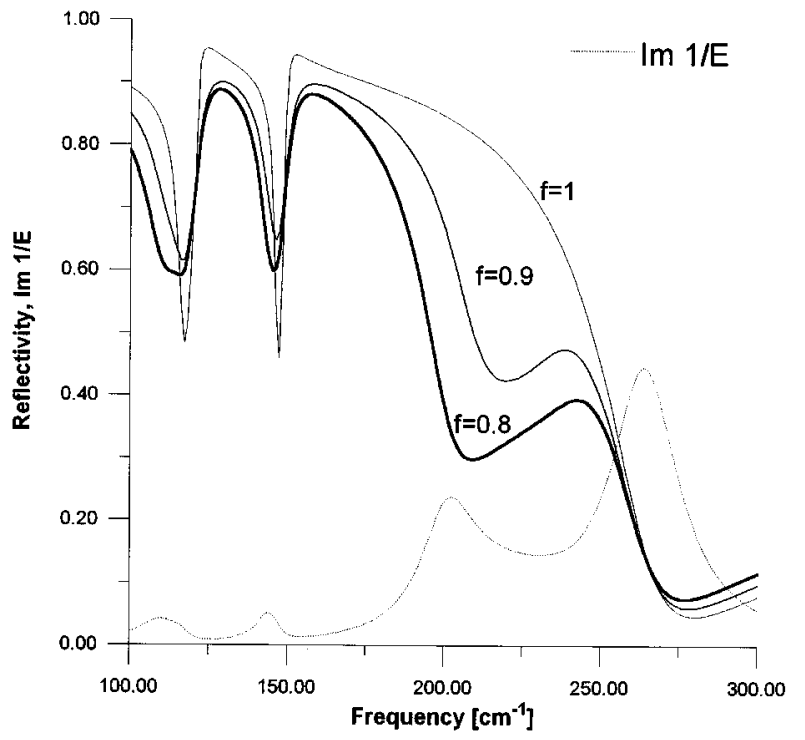

FIG. 9. Normal incidence reflectivity (full curves) for different values of the volume fraction of the metallic component and $\operatorname{Im} \epsilon^{*}$ for $f=0.8$.

$\sqrt{2 / 3} \omega_{p}$ which is rather far from the TO region. Accordingly, we see all three transverse resonances (two TO and MG) practically unchanged.

Since the plasmon frequency for heavily doped CMT falls in the region near the phonon reststrahl band, ${ }^{35}$ $P$-polarization oblique incidence reflectivity and Raman spectra should have dips and peaks, respectively, at the plasma-shifted LO frequencies as well as at the unshifted HgTe-like and CdTe-like LO phonon frequences. In the $D$ regions there is no phonon-plasmon coupling. For the composite system, there are five peaks of $-\operatorname{Im} \epsilon^{*-1}$ correspond-

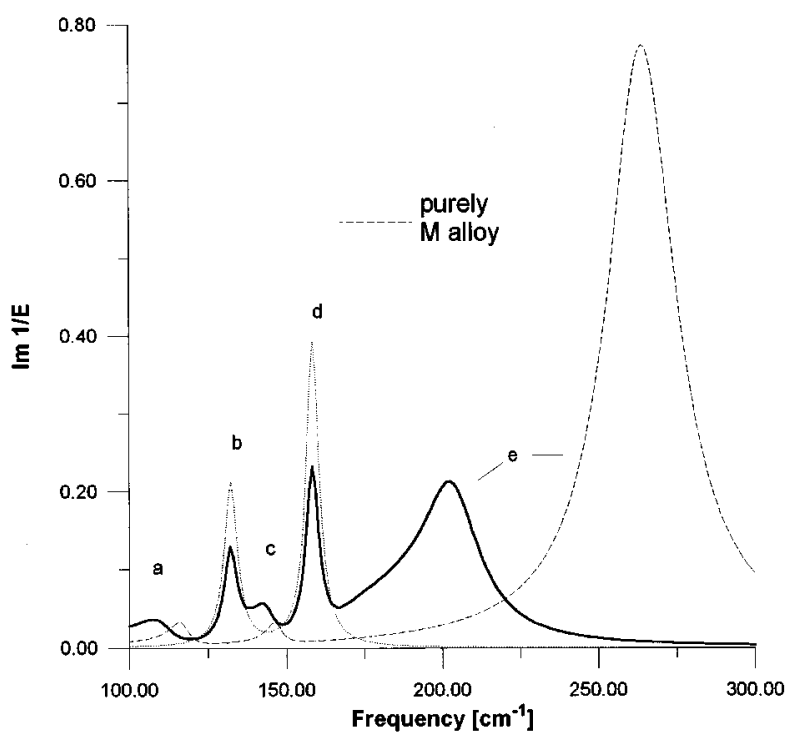

FIG. 10. Imaginary part of $\epsilon^{*}$ for the purely $D$ (dotted curve) and composite alloy containing $f=0.2$ of the metallic inclusions (full curve). Marked features are as follows: $a, c, e$ : mixed LO plasmon modes; $b$ and $d$ : unshifted LO modes. 
ing to both plasmon-phonon and purely LO phonon polaritons (Figs. 9 and 10) which are resolved to a different extent for the parameters used in our calculations. However, in the case of the $M$ inclusions the coupled modes are less shifted from the LO frequencies than if it were in the bulk (especially mode $e$ in Fig. 9), and in the case of the $D$ inclusions purely LO phonon polaritons (modes $b$ and $d$ in Fig. 10) are shifted from the bulk LO phonon frequencies. This is because the inclusions are separated from each other by a medium where the corresponding excitations cannot propagate. We have already noticed this "composite effect" in Sec. III.

\section{DISCUSSION}

The examples considered in Sec. III show that the effect of the dispersion of the sphere's size is small within the renormalized MG approach unless the usual polarizability depends strongly on the sphere's size. This is the case of the electron-hole transitions in SDG, where formula (14) enables one to take proper account of the size dispersion and the dipolar fluctuations in the composite. It should be emphasized that averaging simply the absorption line shapes as is commonly used, ${ }^{7,9,27}$ one considers the spheres as independent of each other; that is the fluctuational interaction between them is neglected. Of course, if the filling fraction is small, the difference is small too, but for $f \sim 0.1$ it should be appreciable.

Formula (14) has been applied with some success to describe the dependence on frequency of the absorption coefficient of spheres embedded in gelatine for $f=0.21$ near the resonant frequency of $\alpha$. We believe that this approach is applicable up to $f \sim 0.3$, i.e., below the percolation threshold.

It is worth comparing $\epsilon^{*}$ given by formulas (1) and (16) to that obtained within SCA by applying the condition of the absence of scattering. In the case of low scattering $\left[f \rightarrow 0,\left(\epsilon_{s}-\epsilon_{h}\right) \rightarrow 0\right]$ both approaches give the same result. ${ }^{23}$ However, if scattering is strong [i.e., $\left(\boldsymbol{\epsilon}_{s}-\boldsymbol{\epsilon}_{h}\right)$ is not small], the two approaches are not equivalent any more. SCA can be applied if $n \alpha$ is not too small, i.e., if the number of scatterers does not fluctuate too strongly. This is known concerning the coherent potential approximation in the theory of alloys ${ }^{36}$ to which SCA is analogous. Thus, DEM and SCA are complementary in the case of strong scattering, which includes all the examples considered in this paper. This statement is illustrated in Fig. 11. The absorption curve for $\mathrm{Cd}_{x} \mathrm{Hg}_{1-x} \mathrm{Te}$ with $f=0.5$ of the metallic inclusions calculated using the SCA approach seems to be quite a reasonable interpolation between the cases of $f=0.2$ and $f=0.8$ treated within DEM. Naturally, all the features are smoothed out in the SCA curve, as is characteristic of any mean-field theory.

The DEM approach, which improves the MG approximation (analogous to the average $T$-matrix approximation in the theory of alloys ${ }^{36}$ ) by including some infinite subsequences of the pertubation theory expansion (5) is not a mean-field theory since it retains the structure of the effective medium: after averaging it still consists of spheres (with renormalized polarizability) embedded in a matrix. Accordingly, this approach gives a richer structure in the spectra. For instance, splitting of the CdTe-like and $\mathrm{HgTe}$-like TO phonon modes in the metallic inclusions of $\mathrm{Cd}_{x} \mathrm{Hg}_{1-x} \mathrm{Te}$ due to their interaction with the MG excitation is clearly seen (Figs. 8 and

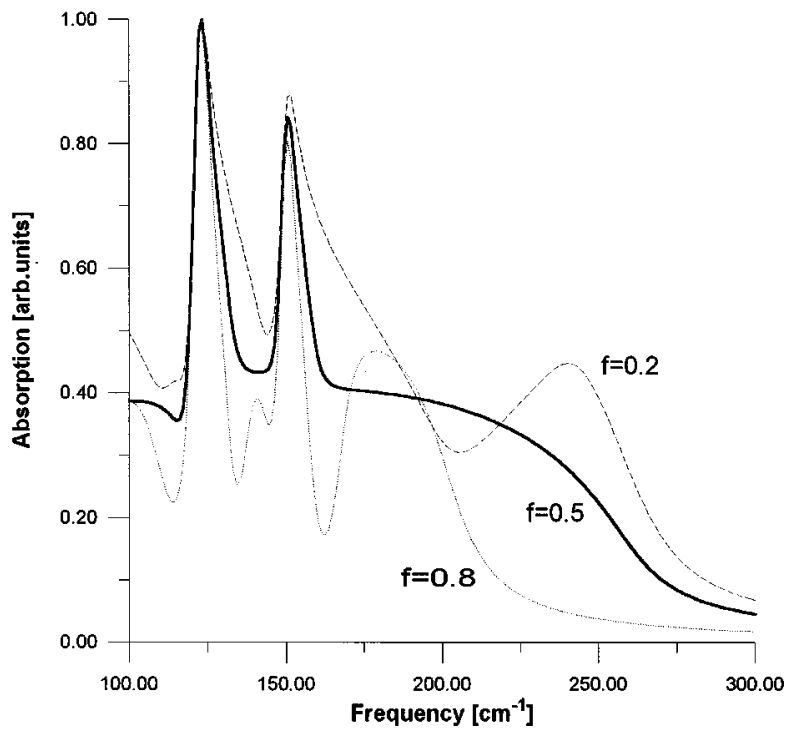

FIG. 11. Comparison of two models FIR absorption of inhomogeneous CMT vs frequency for $f=0.5$ [calculated using formula (23), full curve], $f=0.2$ (dotted curve), and $f=0.8$ (dashed curve).

11). It is also worth mentioning that, within our model, the reflectivity spectra of inhomogeneous $\mathrm{Cd}_{x} \mathrm{Hg}_{1-x} \mathrm{Te}$ should be similar in $S$ and $P$ polarizations. As can be seen from Fig. 9 , each feature in the dependence of $r$ on frequency has a corresponding peak of $\operatorname{Im} \epsilon^{*-1}$ implying a dip in the $P$-polarizaton reflectivity spectrum. Such a similarity of the spectra taken in two polarizations from a $\mathrm{Cd}_{0.65} \mathrm{Hg}_{0.35}$ Te heterostructure grown by low-temperature epitaxy was observed in Ref. 11.

Finally, we would like to make a remark regarding the composite effect pointed out in Sec. III. Due to spatial confinement within the spheres (which has nothing to do with their quantization), transverse and longitudinal excitations are blueshifted and redshifted, respectively. This is analogous to the situation with the phonon modes in pseudobinary polar alloys such as $\mathrm{Cd}_{x} \mathrm{Hg}_{1-x} \mathrm{Te}$. The dependence of the TO and LO CdTe-like and HgTe-like modes on composition is similar to that of CdTe TO and LO phonons in SDG on the filling fraction (Figs. 5 and 6). For alloys, this is known as the two-mode behavior. ${ }^{12,36}$

\section{CONCLUSION}

In this paper, we have derived a formula for renormalized MG polarizability of nonpercolating spherical inclusions in a matrix that simultaneously takes account of dipolar fluctuations due to random positions of the spheres and the effect of the continuous distribution of the sphere's size. Using this formula we have calculated an effective dielectric response for several composite systems. The dispersion of sizes is most important in the case of electron-hole transitions in SDG where inhomogeneous broadening of the optical absorption line shapes is such that just the principal peak can be resolved if $\Delta a / a \sim 10 \%$ for the Gaussian distribution of sizes. We have discussed the shifts of the transverse and longitudinal excitation in the spheres due to their spatial con- 
finement. This effect appears already in the original MG approximation, but the dipolar fluctuations lead to an appreciable additional shift and broadening of the absorption lines. For a $\mathrm{Cd}_{x} \mathrm{Hg}_{1-x} \mathrm{Te}$ alloy with semimetallic inclusions, this leads to an interaction of the (shifted) plasmon excitation with phonons. It would be interesting to revise published experimental reflectivity data taken from this often inhomogeneous material in the view of the presented calculated re- sults. This may help to find evidence for the existence of such inclusions evoked to explain some transport anomalies in $\mathrm{Cd}_{x} \mathrm{Hg}_{1-x} \mathrm{Te}^{5,6}$

\section{ACKNOWLEDGMENTS}

M.I.V. wishes to acknowledge financial support from CAPES (Brazil) and RFFI (Russia).
${ }^{1}$ A. J. R. de Kock, Philos. Res. Rep. (Suppl.) 1, 8 (1973).

${ }^{2}$ A. N. Georgobiani et al., Fiz. Tek. Poluprovodn. 19, 810 (1985) [Sov. Phys. Semicond. 19, 99 (1985)].

${ }^{3}$ R. Sporken, S. Sivananthan, J. Reno, and J. P. Faurie, Phys. Rev. B 38, 1351 (1988).

${ }^{4}$ N. N. Berchenko et al., Pis'ma, Zh. Eksp. Teor. Fiz. 18, 76 (1992) [JETP Lett. 18, 41 (1992)].

${ }^{5}$ M. C. Chen, S. G. Parker, and D. F. Weinrauh, J. Appl. Phys. 58, 3150 (1985).

${ }^{6}$ J. G. Tian et al., Appl. Phys. Lett. 59, 2591 (1991).

${ }^{7}$ W. Y. Wu, J. N. Shulman, T. Y. Hsu, and U. Efron, Appl. Phys. Lett. 51, 710 (1987).

${ }^{8}$ J. A. Medeiros Neto et al., Appl. Phys. Lett. 59, 2715 (1991).

${ }^{9}$ B. G. Potter, J. N. Simmons, P. Kumar, and C. J. Stantor, J. Appl. Phys. 75, 8039 (1994).

${ }^{10}$ I. V. Guzchina et al., Zh. Eks. Teor. Fiz. 106, 1830 (1994) [Sov. Phys. JETP 79, 994 (1994)].

${ }^{11}$ S. K. Kang et al., in Proceedings of the 18th International Conference on Infrared Millimeter Waves, Colchester, UK, SPIE Vol. 2104 (SPIE, Bellingham, 1993), p. 224.

${ }^{12}$ D. W. Taylor, Optical Properties of Mixed Crystals edited by R. J. Elliott (North-Holland, Amsterdam, 1988), pp. 35-130.

${ }^{13}$ L. M. Schwartz, Physica A 207, 131 (1994).

${ }^{14}$ F. Brouers, J. P. Clerc, and G. Giraud, Phys. Rev. B 44, 5299 (1991)

${ }^{15}$ A. Leibbsch and P. V. Gonzalez, Phys. Rev. B 29, 6907 (1984).

${ }^{16}$ R. G. Barrera, G. Monsivais, W. L. Mochan, and E. V. Anda, Phys. Rev. B 39, 9998 (1989).

${ }^{17}$ R. G. Barrera, G. Monsivais, and W. L. Mochan, Phys. Rev. B 38, 5371 (1988).

${ }^{18}$ R. G. Barrera et al., Phys. Rev. B 39, 3522 (1989).
${ }^{19}$ I. I. Fishchuk, J. Phys. Condens. Matter 4, 8045 (1992).

${ }^{20}$ S. A. FitzGerald, T. W. Noh, A. J. Sievers, L. A. Xue, and Y. Tzon, Phys. Rev. B 42, 5469 (1990).

${ }^{21}$ J. C. Maxwell Garnett, Philos. Trans. R. Soc. London 203, 385 (1904).

${ }^{22}$ R. G. Barrera, C. Noguez, and E. V. Anda. J. Chem. Phys. 96, 1574 (1992).

${ }^{23}$ L. D. Landau and E. M. Lifshitz, Electrodynamics of Continious Media (Pergamon, New York, 1977).

${ }^{24}$ T. Matsubara and Y. Toyozava, Prog. Theor. Phys. 26, 739 (1961).

${ }^{25}$ U. Kreibig, A. Althoff, and H. Pressmann, Surf. Sci. 106, 308 (1981).

${ }^{26}$ V. A. Davis and L. Shwartz, Phys. Rev. B 33, 6627 (1936).

${ }^{27}$ Al. L. Efros and A. L. Efros, Fiz. Tekh. Poluprovodn. 16, 1215 (1982) [Sov. Phys. Semicond. 16, 775 (1982)].

${ }^{28}$ W. Hayes and R. Loudon, Scattering of Light by Crystals (Wiley, New York, 1978).

${ }^{29}$ A. M. de Paula et al. (unpublished).

${ }^{30}$ I. M. Lifshitz and V. V. Slyozov, J. Phys. Chem. Solids 19, 35 (1961).

${ }^{31}$ B. G. Potter and J. H. Simmons, J. Appl. Phys. 68, 1218 (1990).

${ }^{32}$ M. C. Klein, F. Hache, D. Ricard, and C. Flytzanis, Phys. Rev. B 42, 11123 (1990).

${ }^{33}$ A. Dal Corso, S. Baroni, R. Resta, and S. de Gironcoli, Phys. Rev. B 47, 3588 (1993).

${ }^{34}$ T. W. Noh and A. J. Sievers, Phys. Rev. Lett. 63, 1800 (1989).

${ }^{35}$ I. M. Tsvidilkovski, G. I. Harus, and N. G. Shelushinina, Adv. Phys. 34, 43 (1995).

${ }^{36}$ R. J. Elliott, J. A. Krumhasl, and P. L. Leath, Rev. Mod. Phys. 46, 465 (1974). 